\title{
Superconducting String Theory
}

\author{
Sergio P. F.
}

Independent Researcher, Madrid, Spain

\section{ABSTRACT}

Gravity explained by a new theory, 'Superconducting String Theory (SST)', inspired on initial string theories and completely opposite from actual fields based. Strengths are decomposed to make strings behave as one-dimensional with universe acting as a superconductor where resistance is near 0 and matter moves inside. Strong nuclear force, with an attraction of 10.000 Newtons is which makes space to curve, generating acceleration. More matter, more acceleration. Electromagnetic moves in 8 decimals, gravity is moved to more than 30 decimals to work as a superconductor.

\section{Indexing terms/Keywords}

superconducting string theory; unified field theory; strong nuclear force; gravity; energetic vacuum; dark matter; relativity; QCD;

\section{Academic Discipline And Sub-Disciplines}

Theoretical Physics;

\section{SUBJECT CLASSIFICATION}

Relativity; Central theories;

\section{TYPE (METHOD/APPROACH)}

Central theories

\section{INTRODUCTION}

The 'Theory of Everything' is a hypothetical theory of physics that explains and connects all known physical phenomena into one. There is a possible solution to the origin of gravity force, postulating it as angular piece of this theory, this solution erases gravity as one of the fundamental forces of nature and unifies it with strong nuclear force.

Let's analyze the forces that occur in the universe transforming string theory. It allows explaining many physical behaviors that without its existence would be practically impossible to understand, even so, these strings have not been able to be discovered and are only that, a theory that serves as an important support to the world of physics. One of the best known theoretical applications about them is how their vibration can provoke the creation of matter, but this is not about theories already written, we are going to place these strings in a simpler way to answer some doubts in subatomic world.

This theory uses 4 dimensions in space and a behavior as one dimension in strings with superconducting capacities. Like an elastic band between V-shaped sticks where the elastic band slides down, the strong nuclear force, forces these strings to bend to fall dawn.

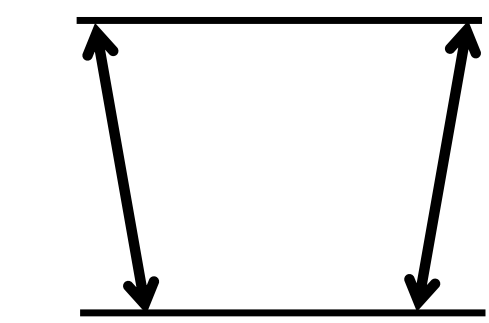

2 strings separated by 180 pixels = 1 space unit example

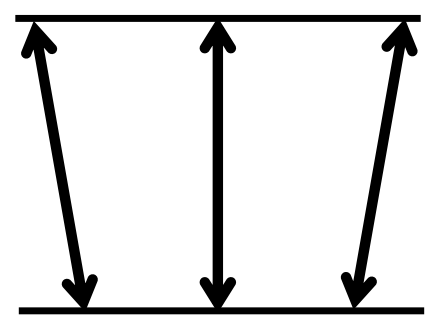

3 strings separated by 90 pixels = 2 space unit's example

Fig. 1: String example.

It's not directly related to electromagnetism. 


\section{ACTORS}

\subsection{String theory}

String theory is a theoretical framework in which the point-like particles of particle physics are replaced by onedimensional objects called strings. Each string that we cross would be the minimum distance that can be traversed during a displacement.

We can note two important qualities of strings:

- Distance to the most distant object detected by the human being is more than 30 billion light years, that means there are beams of light which are able to travel that distance without decreasing its speed (they modify only its wavelength). Like light, an object can move into space for a practically unlimited period, as long as it doesn't find a force to stop it. If strings exist, they act as a superconductor of matter with a resistance near 0 .

- In order to generate waves it's easier into a strongly linked structure. Gravitational waves behave like ocean waves which are similar to an uptight net, these tensions can be decomposed as one-dimensional structure for its study. Strings, at same time, could be one or zero-dimensional, like points under extreme bound forces, think about them as something tenser than any cable that holds the heaviest bridge in the world.

The new framework we have drawn would be a set of extremely tense strings, with a practically infinite matter conduction capacity. Remember we are moving into universe at an estimated speed of $600 \mathrm{~km} / \mathrm{sc}$.

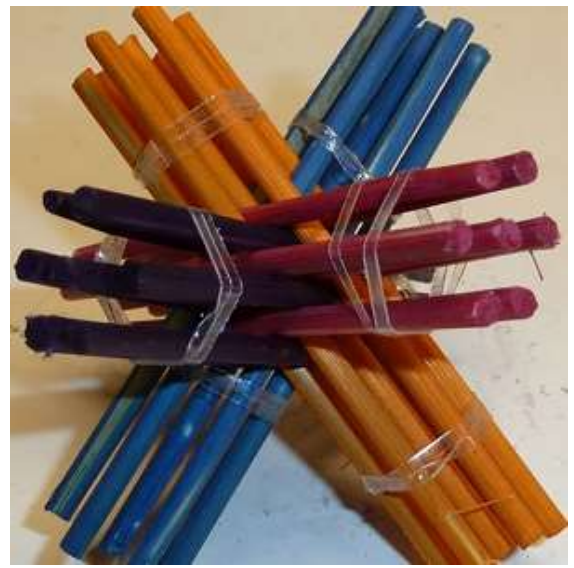

Fig. 2: Example of quantum entanglement. Gravity waves behave as an uptight net.

\subsection{Strong nuclear force}

Strong nuclear force is another variable. This force allows the atomic nucleus to remain together, being the strongest of the so-called fundamental interactions (gravitational, electromagnetic, strong nuclear, and weak nuclear). Gluon is in charge of this interaction, it has a scope not greater than 10 to the power of -15 meters, preventing matter to separate by a constant attraction force between quarks of maximum $10.000 \mathrm{~N}(\mathrm{~F})$.

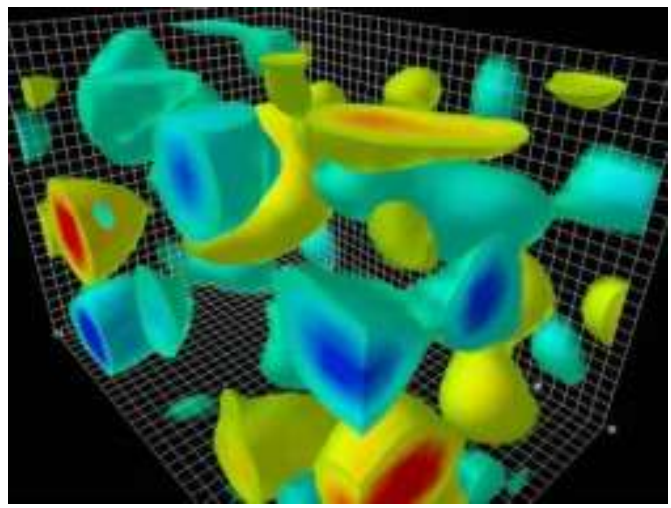

Fig. 3: Quark and gluon interactions into vacuum (Special Research Centre for the Subatomic Structure of Matter). 
This real picture illustrates the three-dimensional structure of gluon-field configurations, describing the vacuum properties. The volume of the box is 2,4 by 2,4 by $3,6 \mathrm{fm}$. Contrary to the concept of an empty vacuum, this induces chromo-electric and chromo-magnetic fields in its lowest energy state. The frame rate into this example is billions of billions frames per second (FPS).

\section{SUPERCONDUCTING STRING THEORY (SST)}

\subsection{Fundamentals}

We have created a scenario with a superconductor of matter interacting with a force that makes that matter hold together, but, how can they interact with each other? The most simple is to think about two V-shaped sticks (simulating the strings), and an elastic band that tight them at the most opened side (it would simulate the gluon, with size 10 to the power of -15 meters). If sticks are sufficiently lubricated and tense, what does the elastic band do? It will slide to the thinnest side. More elastic bands, more force will be exerted on the sticks to join them, so next bands will slide even faster (equally, more mass causes more attraction).

We are talking about unknown limits in known world, such as infinite conduction or tensions never seen in materials.

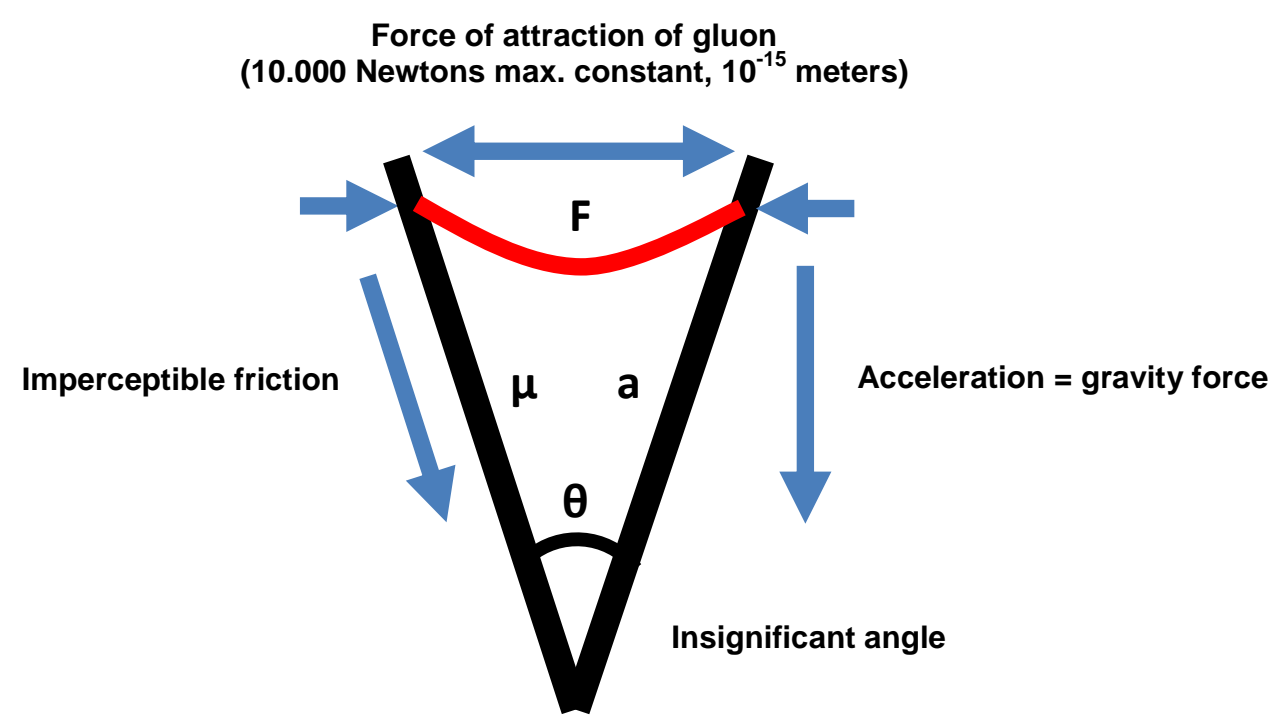

Fig. 4: Implied forces.

Suddenly, we have erased one of the fundamental forces of nature, gravity force doesn't really exist, exists the strong nuclear force interacting with strings. I have called this theory 'Superconducting String Theory (SST)'.

\subsection{Calculations}

Apply formulas from inclined planes (Newton's second law). Simulation is in horizontal direction.

Friction is imperceptible and acceleration down the plane is matched with gravity acceleration in our planet. Vertical force is not gravity force, it is gluon force, which values are estimated, so we keep force $10.000 \mathrm{~N}\left(\mathrm{~F}_{1}\right)$ and mass of 0,0002 $\mathrm{eV} / \mathrm{c}^{2}\left(\mathrm{~m}_{2}\right)$. It can be considered vertical angle, but it's depreciable. 
Acceleration now is gravity, gluon force acts as vertical attraction

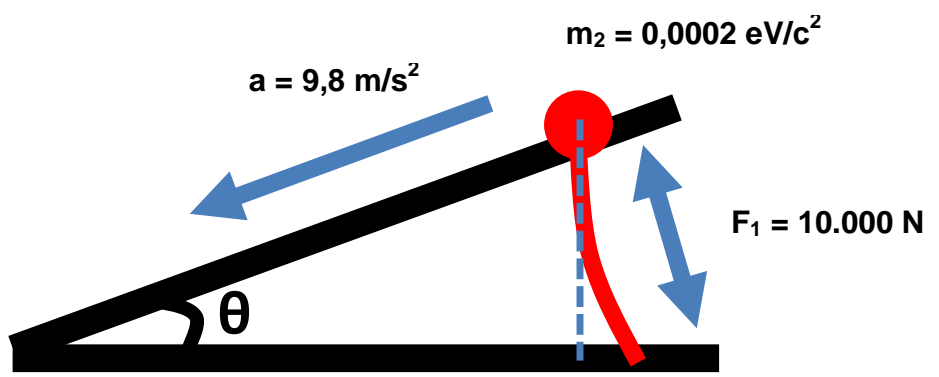

Fig. 5: Inclined plane forces (gluon catching strings).

$m_{1} \times g \times \operatorname{sen}(x)=m_{2} \times a$

Match vertical force $\left(F_{1}\right)$ with the attraction force from one gluon (estimated as a constant with max. 10.000 N).

$F_{1}=m_{1} \times g=10.000 \mathrm{~N}$

$F_{1} \times \operatorname{sen}(x)=m_{2} \times a$

Convert variables to metric system considering the mass of the gluon $\left(0,0002 \mathrm{eV} / \mathrm{c}^{2}\right)$.

$1 \mathrm{eV} / \mathrm{c}^{2}=1,782662 \times 10^{-36} \mathrm{~kg}$

$m_{2}=0,0002 \times 1,782662 \times 10^{-36}=3,565324 \times 10^{-40} \mathrm{~kg}$

$a=9,8 \mathrm{~m} / \mathrm{s}^{2}$

$F_{2}=m_{2} \times a=3,565324 \times 10^{-40} \times 9,8=3,49401752 \times 10^{-39} \mathrm{~N}$

Angle calculation from a cluster of strings under gluon influence.

$x=\operatorname{arcsen}\left(F_{2} / F_{1}\right)$

$F_{2} / F_{1}=3,49401752 \times 10^{-43} \mathrm{~N}$

$x=\operatorname{arcsen}\left(3,49401752 \times 10^{-43}\right)=3,49401752 \times 10^{-43}$ 。

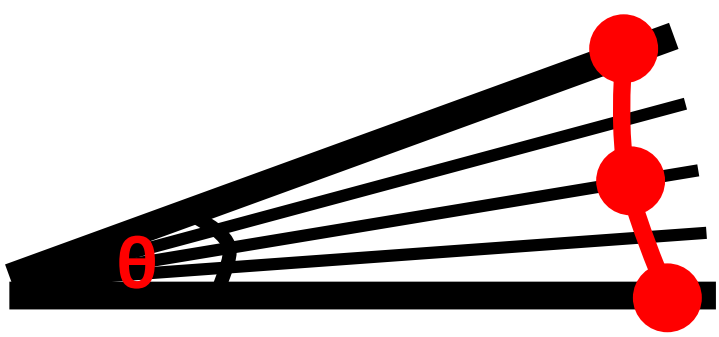

Fig. 6: Gluon scope catches more than one string.

\section{CONCLUSIONS}

It can explain:

- What is gravity force?

- Unified field theory between gravity and strong nuclear force.

- New behavior in dark matter because of differences in density of the superconductor. Lower density generates bigger angle, this implies bigger attraction force. If threads are separated, matter $\left(m_{2}\right)$ becomes energy $\left(F_{1}\right)$. Some places at universe could have bigger accelerations because of this effect; this means much less dark matter (which is estimated at $27 \%$ of the mass and energy in the observable universe). 


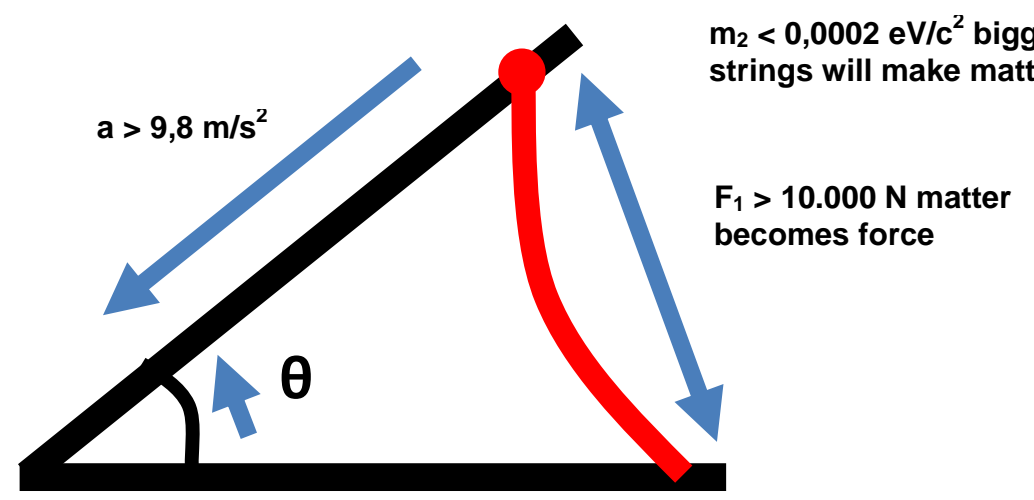

Fig. 7: Increase the separation, increase the force exerted. Matter is transformed into energy.

$F_{1} \times \operatorname{sen}(x)=m_{2} \times a$

$1 \mathrm{eV} / \mathrm{c}^{2}=1,782662 \times 10^{-36} \mathrm{~kg}$

$m_{2}=0,0002 \times 1,782662 \times 10^{-36}=3,565324 \times 10^{-40} \mathrm{~kg}$

$F_{2}=m_{2} \times a=3,565324 \times 10^{-40} \times a\left(\mathrm{~kg} /\left(\mathrm{m} / \mathrm{s}^{2}\right)\right)$

Calculate acceleration in relation to angle.

$x=\operatorname{arcsen}\left(F_{2} / F_{1}\right)$

$F_{2} / F_{1}=\left(3,565324 \times 10^{-40} \times a\right) / 10.000=\left(3,565324 \times 10^{-44} \times a\right)$

$x=\operatorname{arcsen}\left(3,565324 \times 10^{-44} \times a\right)=\left(3,565324 \times 10^{-44} \times a\right)^{\circ}$

Bigger angle generates more acceleration.

$a=\left(x / 3,565324 \times 10^{-44}\right) \mathrm{m} / \mathrm{sg}^{2}$

- $\quad$ Einstein field equations. Apply Hooke's law to gluon force as a vertical spring to calculate tensions.

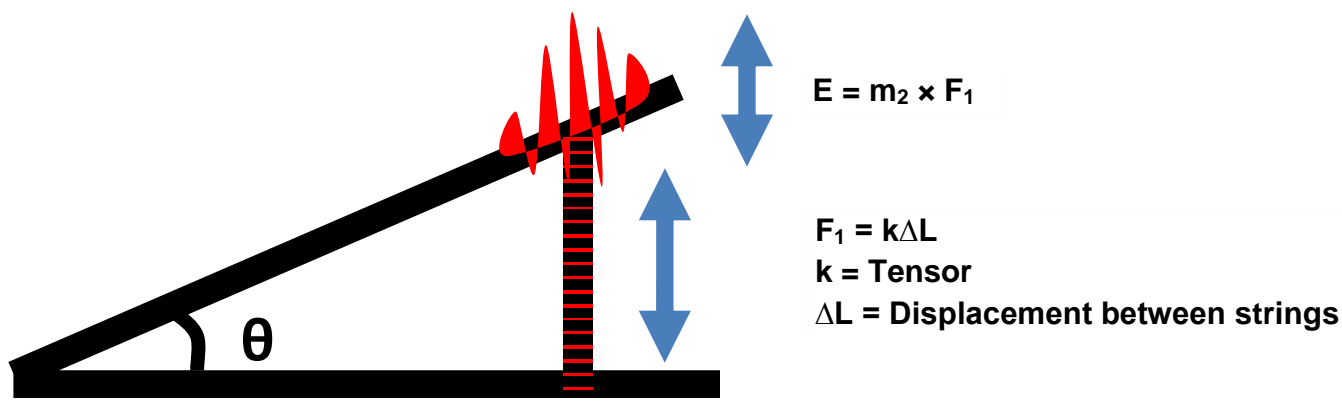

Fig. 8: Energy doesn't disappear, becomes matter.

Gluon force has negative correlation when transforming into matter, decrease $F_{1}$ or increase $m_{2}$ implies less acceleration.

$a=F_{1} \times \operatorname{sen}(x) / m_{2}$

$a=5.000 \times \operatorname{sen}\left(3,49401752 \times 10^{-43}\right) / 3,565324 \times 10^{-40}$

$a=4,9 \mathrm{~m} / \mathrm{s}^{2}$

- $\quad$ Spherical and circular movements at planets and galaxies. Space deforms not proportionally to create more acceleration near accumulation of matter, behaving like an elastic material; this behavior can be quantified by Young's modulus which represents the factor of proportionality in Hooke's law at non-linear systems. 

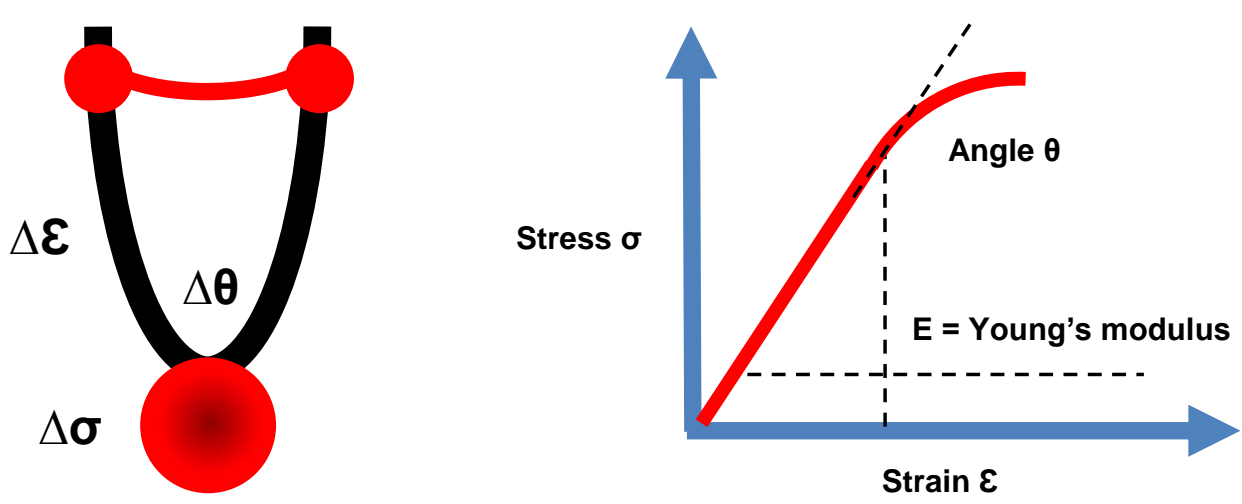

Fig. 9: Angle originated by big amounts of matter has an interaction over very large distances.

The elastic modulus or Young's modulus (E) depends on the force exerted by matter $(\sigma)$ and the deformation at each point of the resulting vector $(\varepsilon)$.

$E=\Delta \sigma / \Delta \varepsilon$

Force exerted by the angle $(F \theta)$, increases $(\Delta)$ faster than force exerted by gluon $\left(F_{1}\right)$ and its relation with matter $\left(m_{2}\right)$.

$\Delta F \theta>\Delta F_{1} / \Delta m_{2}$

This gluon-matter relation also modifies the density of the superconductor in space, since it induces their approach, therefore we can speak of the existence of a bulk modulus (K).

$K=-V(\Delta p / \Delta V)$

The bulk modulus $(K)$ depends on pressure changes $(p)$ and volume $(V)$; other properties as volume viscosity also called bulk viscosity can be applied.

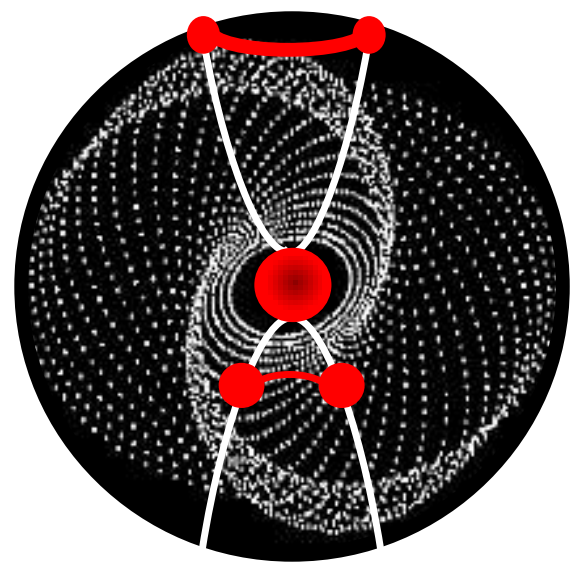

Fig. 10: All these variables help to create shapes in galaxies like the golden spiral $(\varphi=1,6180)$.

- Black holes. Matter and vacuum concentration become strings so close that the scope of the strong nuclear force can be weaker to create a gravitational force; photons are attracted because its field can interact with strings but particles like neutrinos can escape.

- Dark energy and universe's expansion. The behavior of the strings imply to have any kind of spin-polarization to expand, at least, strong enough to avoid get closer and reestablish its structure after any contraction. This strength propagates over long distances.

- Gravitational time dilation. Frames are composed by sets of strings which can deform, when vacuum expands, the points of contact becomes smaller, therefore, if the speed around each points is faster and the speed at each point of contact is smaller, time to cross a bigger frame can become shorter. 


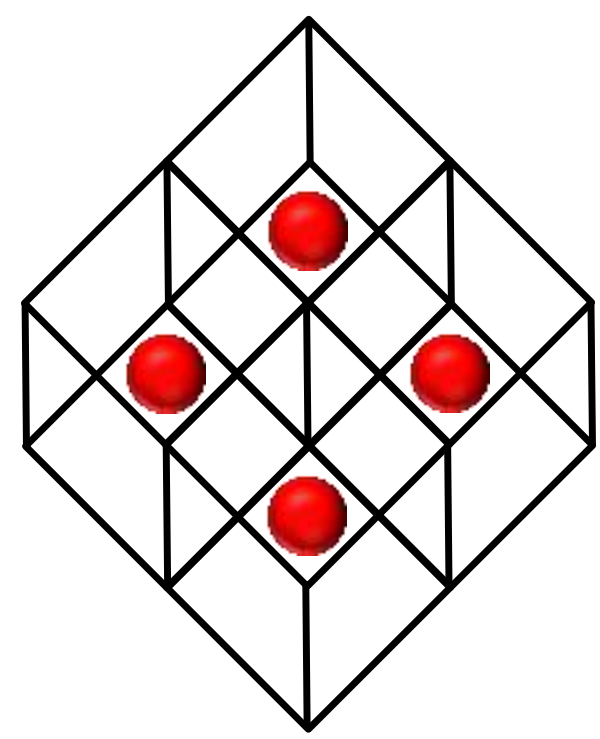

Less gravity, bigger frame, displacement takes less time

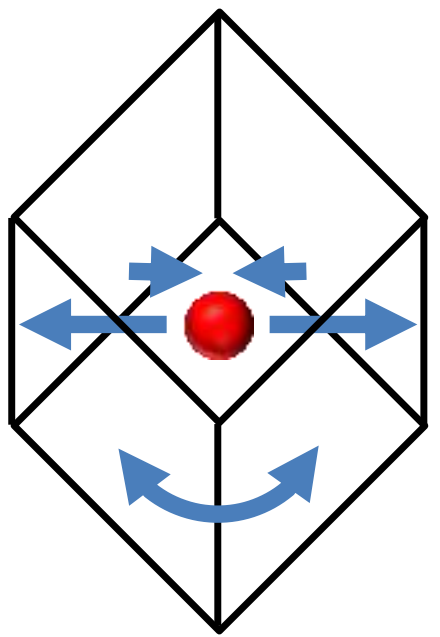

Fig. 11: Vacuum variations in proportions, dimensions and angles are expected to align.

- Planck length $\left(\ell_{P}=1,616229(38) \times 10^{-35} \mathrm{~m}\right)$ which is theoretically considered to be the quantization of space and can indicate the distance between strings.

- Gravitational constant $\left(\mathrm{G}=6,67408(31) \times 10^{-11} \mathrm{~m}^{3} \mathrm{~kg}^{-1} \mathrm{~s}^{-2}\right)$ and its problem to measure with high accuracy since it can be related to the density exposed.

- Schrödinger equation, to describe how the quantum state changes similar to Newton's second law. Leptons are affected by gravity too, so the wavelength of an electron should be able to interact with strings.

- Cosmic Inflation and conservation of angular momentum at rotations into space.

- $\quad$ Gluon size and its larger size far from earth.

\section{MODEL}

\subsection{Fundamental interactions}

The result of SST unification is a new model to represent fundamental interactions:

- Electromagnetic and weak force are unified by the electroweak interaction.

- $\quad$ Strong force and gravity have been unified by Superconducting String Theory (SST).

- Energetic vacuum is considered a new fundamental interaction because it doesn't appear to be reducible to more basic forces.

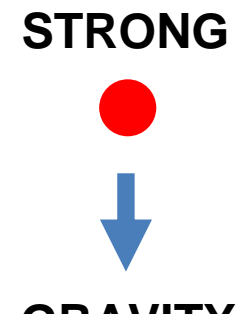

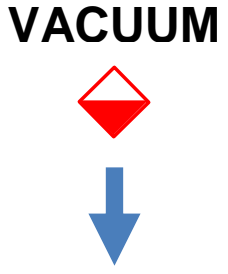

MOTION

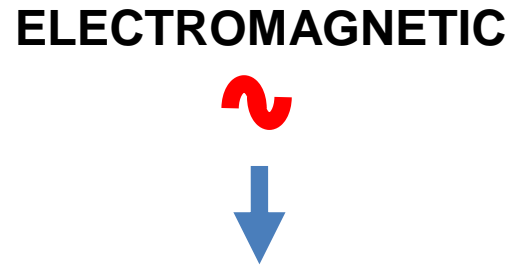

WEAK

Fig. 12: Three basic forces, strong, vacuum and electromagnetic. 


\subsection{Theory of everything}

Motion is needed to unify fundamental forces. Theory of everything (ToE) could be related with movement inside an energetic vacuum where baryonic particles are best candidates to be conducted through it. Behaviors like time, molecular interactions, spin, particles decay or even electromagnetism, could be related with different types of mediation between particles and vacuum.

On this basis, it's possible to deduce some new behaviors. Energetic vacuum and motion would be involved in the generation of the three QCD colors and anticolors, so best structure for vacuum is three fixed polarized directions.
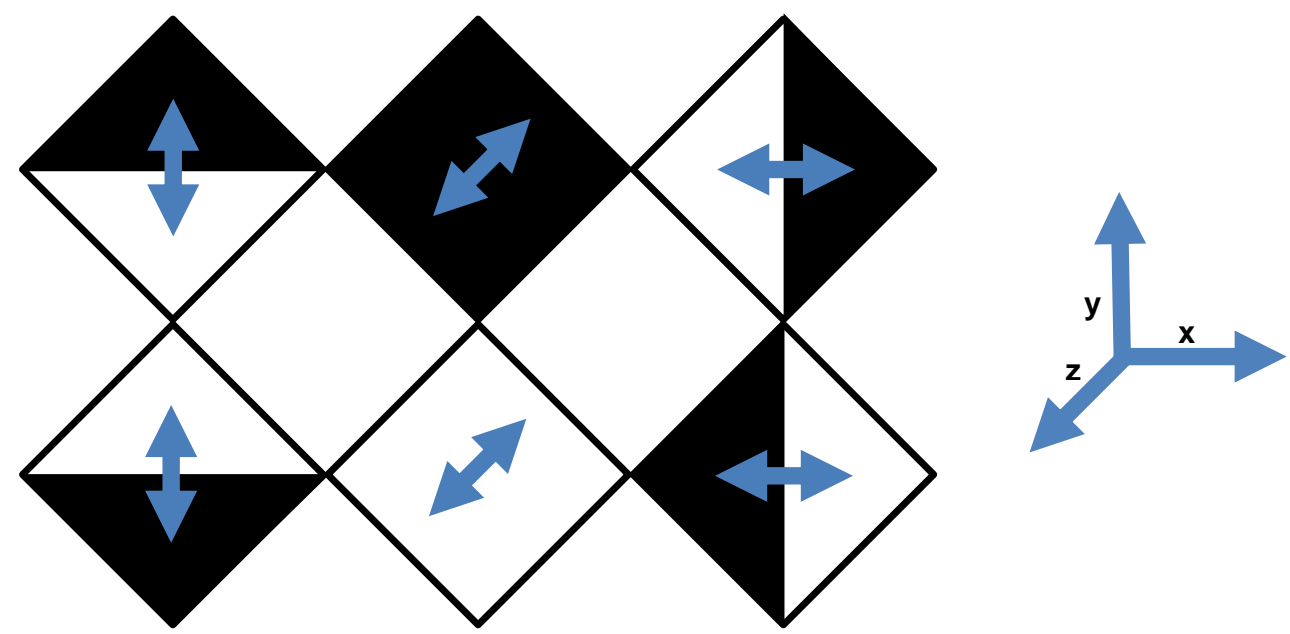

Fig. 13: Vacuum polarization affecting spin interactions have been reported.

Other relations can be found, Coulomb's and Newton's law and their respective constants, would be connected by differences in the forces exerted between baryons and leptons with strings; electromagnetic waves propagation would be connected with vacuum states.

\section{CONSIDERATIONS FOR THE STUDY}

Accelerate matter using a cyclotron to transform into energy so much matter as possible. We need a material with maximum magnetic permeability on high magnetic fields as possible, pure iron can be a good reference but we can consider some other materials with high permeability.

Particles reach their maximum energy at the periphery of the disc $(v=q B R / m)$, the output energy can be calculated when a speed is reached in a relativistic approximation.

$E=1 / 2 m v^{2}=q 2 B 2 R 2 / 2 m$

Centripetal force, forces matter to the sides, so we need a magnetic field to keep dimensions. We need enough width to study how strings bend and enough height to concentrate energy along (it is very difficult to concentrate kinetic energy at one point).
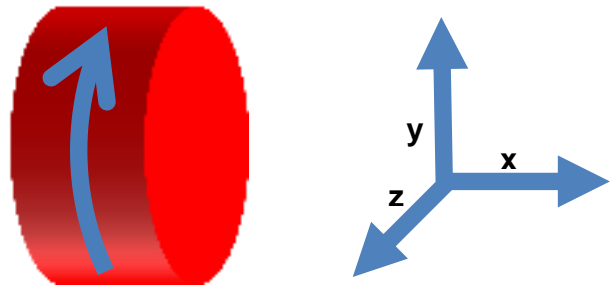

Fig. 14: Accelerate cylinder particles until 1/10 speed of light.

Calculate the energy of one disc in movement, a radius of $2 \mathrm{~cm}$ and a height of $2 \mathrm{~cm}$ are enough to study. 


$$
\begin{aligned}
& V=\pi \times r^{2} \times h \\
& V=3,1416 \times 4 \times 2 \\
& V=25,1328 \mathrm{~cm}^{3}
\end{aligned}
$$

Calculate the mass using iron density $(\rho=7,874 \mathrm{gr} / \mathrm{cm} 3)$ and consider a maximum speed reached.

$m=25,1328 \times 7,874=197,89 \mathrm{gr}=0,197 \mathrm{~kg}$

$v=300.000 \mathrm{~km} / \mathrm{sg}=3 \times 10^{7} \mathrm{~m} / \mathrm{sg}$

We need all energy at vertical to obtain significant values

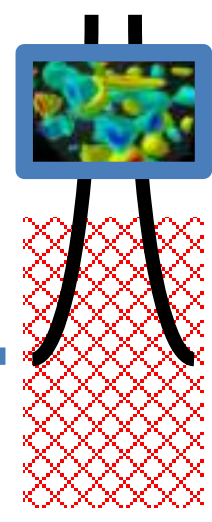

Vacuum density changes

Energy density can be increased about $1 / 100$ at a speed of $3 \times 10^{7} \mathrm{~m} / \mathrm{sg}$

\section{Fig. 15: Energy avoids strings to penetrate.}

Compare kinetic energy reached with the maximum energy which could be generated using a relativistic approximation.

$E_{k}=1 / 2 m v^{2}$

$E_{k}=1 / 2 \times 0,197 \times 9 \times 10^{14}$

$E_{k}=0,8865 \times 10^{14}$

$E=m c^{2}$

$E=0,197 \times 9 \times 10^{16}$

$E=1,773 \times 10^{16}$

Other variations at QCD have been observed, for example, at baryon resonances.

\section{REFERENCES}

1. Daniel Baumann \& Liam McAllister (2015). Inflation and String Theory. Cambridge Monographs on Mathematical Physics, ISBN 978-1316105733. https://doi.org/10.1017/CBO9781316105733

2. Henning Genz (1999). Nothingness: The Science of Empty Space. Addison-Wesley. ISBN 978-0201442335.

3. Bechler, Z. (2012). Newton's Physics and the Conceptual Structure of the Scientific Revolution. Springer Science \& Business Media. http://dx.doi.org/10.1007/978-94-011-3276-3

4. Gould, P. H. (2013). Introduction to Linear Elasticity. http://dx.doi.org/10.1007/978-1-4614-4833-4

5. Cannon, J. T., \& Dostrovsky, S. (1975). The Evolution of Dynamics: Vibration Theory from 1687 to 1742. http://dx.doi.org/10.1007/978-1-4613-9461-7

6. F. Yndurain (1995). Limits on the mass of the gluon. Physics Letters B. 345 (4): 524. http://dx.doi.org/10.1016/0370-2693(94)01677-5

7. Williams, A. G., Bonnet, F. D., Bowman, P. O., Leinweber, D. B., Skullerud, J. I., \& Zanotti, J. M. (2002). The Transition from Nonperturbative to Perturbative QCD. In Non-Perturbative QCD (pp. 189-196). http://dx.doi.org/10.1142/9789812778352_0026

8. J. Li, H. Y. Guo, B. N. Wan, X. Z. Gong, Y. F. Liang, G. S. Xu, K. F. Gan, J. S. Hu, H. Q. Wang, L. Wang, L. Zeng, Y. P. Zhao, P. Denner, G. L. Jackson, A. Loarte, R. Maingi, J. E. Menard, M. Rack \& X. L. Zou (2013). A long-pulse high-confinement plasma regime in the Experimental Advanced Superconducting Tokamak. Nature Physics 9, 817-821. http://dx.doi.org/10.1038/nphys2795 
9. Andreas S. Kronfeld \& Chris Quigg (2010). Resource Letter QCD-1: Quantum chromodynamics. http://dx.doi.org/10.1119/1.3454865

10. Milgrom, M. (1983). A modification of the Newtonian dynamics as a possible alternative to the hidden mass hypothesis. Astrophysical Journal. 270: 365-370. http://dx.doi.org/10.1086/161130

11. M.A. Shifman, .A.I. Vainshtein,V.I. Zakharov (1979). QCD and resonance physics. Theoretical foundations. http://www.sciencedirect.com/science/article/pii/0550321379900221

\section{Author' biography}

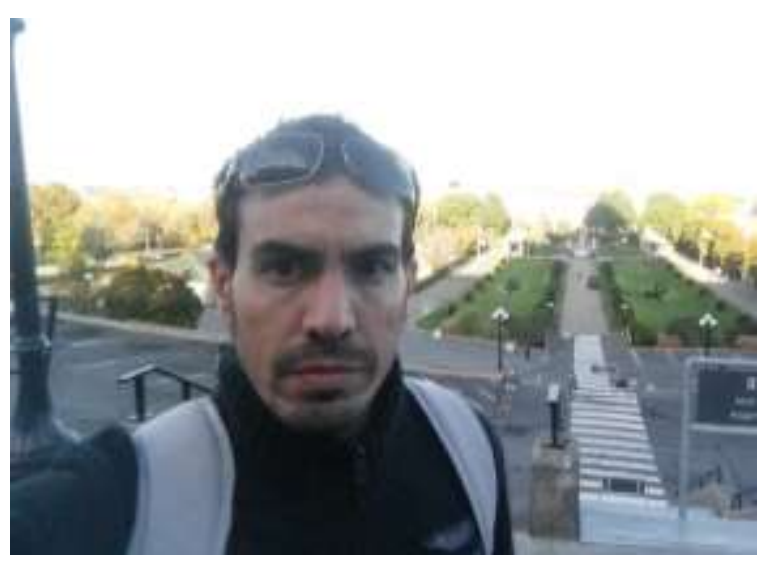

Sergio Pérez Felipe was born in León, Spain in 1979.

He graduated from Computer Science.

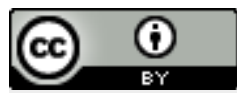

This work is licensed under a Creative Commons Attribution 4.0 International License. 